\title{
Evaluation of the potential of nanoparticles containing active substances in selected chronic diseases
}

\author{
Beata Sarecka-Hujarr ${ }^{1, A-F}$, Anna Banyś2,A-F, Aneta 0stróżka-Cieślik ${ }^{2, A-F}$, Radosław Balwierz 3 ,B,D,F, Barbara Dolińska ${ }^{2, D, F}$ \\ ${ }^{1}$ Department of Basic Biomedical Science, Medical University of Silesia in Katowice, Poland \\ 2 Department of Pharmaceutical Technology, Medical University of Silesia in Katowice, Poland \\ ${ }^{3}$ Department of Health Care, Silesian Medical College, Katowice, Poland \\ A - research concept and design; $\mathrm{B}$ - collection and/or assembly of data; $\mathrm{C}$ - data analysis and interpretation; \\ $\mathrm{D}$ - writing the article; $\mathrm{E}$ - critical revision of the article; $\mathrm{F}$ - final approval of the article
}

\section{Address for correspondence}

Beata Sarecka-Hujar

E-mail: bsarecka-hujar@sum.edu.pl

Funding sources

None declared

Conflict of interest

None declared

Received on March 27, 2019

Reviewed on October 28, 2019

Accepted on December 2, 2019

Published online on March 26, 2020

\begin{abstract}
Currently, over $80 \%$ of all deaths result from the incidence of chronic diseases. The challenge of modern medicine is to develop innovative and effective methods of diagnosis and therapy of these disorders. Different types of particles can be obtained with the use of nanotechnology, including nanoliposomes, solid lipid nanoparticles (SLN), nanospheres, dendrimers, as well as carbon nanotubes (CNT) or fullerenes. All of these nanoparticles (NPs) are suggested to have potential, both in medicine and in diagnosis of many diseases, giving a chance for recovery or longer life for the patients. The studies concerning the usage of NPs show their effective role in most cases. However, there are also concerns about their toxicity or long-term adverse effects. The aim of this literature review was to discuss the results of the latest available studies concerning the efficacy of selected drug-loaded nanocarriers in several chronic diseases, i.e., cardiac disorders, cancer, Alzheimer's disease (AD), Parkinson's disease (PD), and wound healing. We also focused our attention on the methodology of NPs preparation, materials used for their preparation as well as on positive and negative aspects of these nanocarriers.
\end{abstract}

Key words: cancer, cardiac diseases, nanoparticles, neurodegenerative diseases, wound healing
Cite as

Sarecka-Hujar B, Banyś A, Ostróżka-Cieślik A, Balwierz R, Dolińska $B$. Evaluation of the potential of nanoparticles containing active substances in selected chronic diseases. Adv Clin Exp Med. 2020;29(3):385-397.

doi:10.17219/acem/115005

DOI

10.17219/acem/115005

Copyright

Copyright by Author(s)

This is an article distributed under the terms of the

Creative Commons Attribution 3.0 Unported (CC BY 3.0)

(https://creativecommons.org/licenses/by/3.0/) 


\section{Introduction}

In recent decades, the average life expectancy has significantly increased. Advances in medicine and the improvement of living conditions have significantly reduced mortality caused by infectious diseases and inflammatory conditions of various etiologies. Currently, over $80 \%$ of all deaths are a result of chronic diseases. ${ }^{1}$ The challenge of modern medicine is to develop innovative and effective methods of diagnosis and therapy for chronic conditions. Currently, modern controlled drug release systems are being extensively studied. ${ }^{2}$ However, one of the important issues in the process of developing new drugs is the bioavailability of active pharmaceutical ingredient (API). To overcome this potential problem, several methods can be used, including micro- and nanonization, which can help reduce a substance to a micro- or nanosize without changing its chemical properties. They also increase the surface area of drug particles, especially in the case of nanoparticles (NPs), leading to faster dissolution rates and increased bioavailability in the body. ${ }^{3}$

The use of NPs containing various active substances is widely analyzed in the areas of diagnostics, medicine and pharmacy. Nanoparticles included in the diagnostic tests can help in quick determining the patient's disease state as well as precise indicating the location of the pathological cells in the human body. ${ }^{4}$ Nanoscale methods used in pharmacy make it possible to manufacture spherical- or fibrous-shaped, and single- or multilayered NPs. The new drug delivery systems (DDS) enable API to reach the target site. In addition, the structure of API can be modified. Due to the size of NPs (ranging from $1 \mathrm{~nm}$ to $100-200 \mathrm{~nm}$ ) it is possible to suspend them in a gas and thereby to obtain a therapeutic aerosol. ${ }^{5}$ The NPs can also be suspended in a liquid or embedded into a solid matrix. ${ }^{6}$ The specificity of the nanopreparation is associated with a reduced dose or concentration of API. In addition, reduced adverse effects of API can also be observed. Currently, intensive work is being conducted on biodegradable polymers and natural biopolymers, used in the production of NPs, which will be compatible with the cells of the human body. ${ }^{7}$ It is important to improve technology for the manufacture of NPs; obtained NPs should be characterized by high homogeneity, monodispersity and, thus, optimal efficiency. Nanoscale techniques currently in use have many disadvantages, among which are difficulties in obtaining a product with high stability and low toxicity. During the manufacturing process, some polymorphic transformations can possibly appear, as well as a tendency towards aggregation. In addition, these techniques are time-consuming and require high financial outlays. The results of experimental studies suggest, however, that the use of NPs in medicine may revolutionize the therapeutic success of treating many diseases, i.e., neoplastic diseases, heart diseases, but also neurodegenerative diseases or wounds difficult to heal. ${ }^{7-11}$ On the other hand, controversies about their long-term toxicity, which is not yet fully known, have also been raised. ${ }^{12}$ These concerns are the reason why work is being done to develop the legal regulations regarding nanotechnology methods. ${ }^{13}$ At present, the so-called soft international law regulates the safety of using innovative applications of nanotechnology, which includes recommendations with regard to current standards. ${ }^{14}$

The aim of this literature review was to discuss the results of the latest available studies concerning the efficacy of certain drug-loaded nanocarriers in selected chronic diseases, with special emphasis on methodology as well as materials used during their preparation.

\section{Characteristics of selected drug nanocarriers}

The following types of NPs can be distinguished in relation to the material used: lipid-based (e.g., nanoliposomes, lipospheres, including solid lipid NPs (SLN) and nanostructured lipid carriers), polymeric-based (e.g., nanospheres, nanocapsules) or carbon-based (fullerenes, carbon dots, carbon nanotubes (CNT)). ${ }^{6}$

Nanoliposomes are spherical structures up to 100$200 \mathrm{~nm}$ in size, with the walls formed by a lipid bilayer with an aqueous phase as a core of a vesicle. The structure of the liposomes/nanoliposomes, as well as methods of their preparation and division, are well-understood and have been described in previous studies. ${ }^{6}$ Nanoliposomes show a number of advantages, e.g., lack of toxicity and immunogenicity, full biodegradability and structural versatility as carriers. However, a number of disadvantages of liposomal carriers can be noted. Studies demonstrated, among others, low stability, especially in the case of aquatic environment, high accumulation in the liver and spleen, difficulty in overcoming the blood-tissue barrier, oxidation of the lipid, hydrolysis of the ester moiety, or API release to the external phase. ${ }^{6,15}$ Many studies have, therefore, been performed to modify the structure of liposomes sufficient to obtain the efficient transport of active substances. ${ }^{15,16}$ Nanoliposomes can be often coated with polyethylene glycol (PEG), which may, among other things, affect the half-life of the drug-molecule-transporter conjugate in the blood. ${ }^{16}$

Another spherical lipid-based particles are lipospheres with a size of up to $500 \mathrm{~nm}$, in which 2 types can be distinguished: SLN and nanostructured lipid carriers (NLC). ${ }^{6}$ The structure of SLN resembles the oil/water (o/w) emulsion, in which the liquid lipid has been replaced by a lipid solid at room temperature. ${ }^{17}$ The biologically active compounds are arranged in an aqueous phase containing surfactants between the chains of the hydrophobic compounds. The lipid content in the lipospheres is approx. $0.1-30 \%$ and leads to good in vivo tolerability and reduced toxicity. ${ }^{18}$ Solid lipid nanoparticles provide better stability for encapsulated proteins, preventing their proteolytic 
degradation, in addition to their prolonged release. In contrast with SLN, the matrix in NLC can form both solid and liquid lipids (e.g., Miglyol, Capmul, MCM). ${ }^{19}$ Nanostructured lipid carriers can be manufactured in high-pressure hot homogenization in the form of multiple systems such as oil/solid lipid/water suspension. A dispersing method using a high-speed mixer and a method for emulsion formation and emulsification with evaporation or diffusion of an organic solvent can also be used for the preparation of lipospheres. ${ }^{20,21}$ Both SLN and NLC may provide better stability of the drug, and a reduction of the first-pass-effect after oral administration, as well as mask its bitter taste. ${ }^{21}$

Among polymer-based NPs, nanospheres, nanocapsules and dendrimers are the most studied in the context of drug delivery. ${ }^{6,22-24}$ The polymers used to manufacture these particles should be biodegradable and biocompatible. Simultaneously, the drug should be released at the target site and at a concentration within the therapeutic range. In nanospheres, the active ingredient is incorporated (suspended) in a biodegradable polymer matrix. The mechanism of API degradation is chemical (polymer hydrolysis) and physical as well (matrix erosion). The size of nanospheres (10-200 nm) allows for their free penetration into tissues and cells and prevents them from being absorbed by the cells of the immune system of the body. ${ }^{6}$ Nanocapsules are in the form of colloidal nanovesicles sized $100-500 \mathrm{~nm}$. The core of this structure $(\mathrm{o} / \mathrm{w})$ is surrounded by a polymer membrane. ${ }^{22}$ Synthetic (e.g., polycaprolactone, polyacrylamide, methyl polymethacrylate) and natural (e.g., gelatine, chitosan, albumins) polymers are most commonly used to obtain nanocapsules.

In turn, dendrimers are organic polymer compounds with a highly branched structure in the shape of a sphere. These kinds of NPs consist of the core and radially branching dendrons. Their unique trait is that they can transport the drug in 2 ways: via covalently linking the drug molecules to surface groups of the NPs and by encapsulating the drug in the spaces between the branches of the polymer. In addition, in anticancer therapy these NPs may carry many other substances apart from the anticancer drugs, including fluorescent markers, photosensitizers or monoclonal antibodies. ${ }^{23,24}$

In the field of carbon-based NPs, the best-known and most analyzed are fullerenes, CNT, dots, and nanodiamonds. Fullerenes are an allotropic form of pure carbon with a diameter of approx. $1 \mathrm{~nm}$. The fullerene C60 achieves the fullest chemical stability and high symmetry. These particles are obtained using an electric or laser arc technique. To use fullerenes for therapeutic purposes, their interiors, as well as their surface, can be modified (e.g., through coating with polyvinylpyrrolidone (PVP), by PEG addition or by complexes with cyclodextrins). The following types of fullerenes may be distinguished: endohedral, heterofullerenes and exohedral. Other atoms or molecules can be placed in the middle of the endohedral fullerenes (e.g., metals, lanthanides carbides). In turn, in heterofullerenes, the carbon atoms may be partially or completely replaced by other atoms while exohedral ones can be obtained by surface modifications. Fullerenes show strong antioxidant and protective properties. ${ }^{25}$ This is primarily due to the presence of conjugated double bonds and an unfilled molecular orbital. They are characterized by a high ability to attach free oxygen radicals, so-called radical sponges. ${ }^{26}$

Carbon nanotubes are cylindrical graphene films which are open or closed on one or two sides, with carbon atoms arranged in hexagonal structures. These NPs exhibit many excellent properties, including electronic, thermal and mechanical features. The following types of CNT, due to the number of layers they form, may be distinguished: single-walled CNT (SWCNT), double-walled CNT (DWCNT) and multi-walled CNT (MWCNT). To obtain SWCNT and MWCNT, electric arc discharge methods, laser graphite evaporation and chemical vapor deposition are used. In turn, double-walled nanotubes may be obtained with the encapsulation of $\mathrm{C} 60$ fullerenes inside the single-walled carbon nanotubes. ${ }^{27}$ As with fullerenes, the surface of CNT may be modified. Previous studies have shown that CNT can penetrate cell membranes and are effective carriers of various molecules, including peptides, proteins, plasmids, nucleic acids, and chemotherapeutics. ${ }^{28,29}$ Simultaneously, low toxicity of CNT as well as structures displaying a lack of immunogenicity were also observed. ${ }^{28,29}$ On the other hand, with regard to the safety of using CNT, some data revealed that their toxicity was comparable with asbestos fibers, which induced some pro-inflammatory mechanisms, among others, the secretion of interleukin $1 \beta$ (IL-1 $1 \beta$ ) from lipopolysaccharide (LPS)-primed macrophages. ${ }^{30}$ It was also suggested that, much like asbestos, CNT may cause genotoxic effects. ${ }^{31}$

Table 1 summarizes the characteristics of selected types of NPs (description of their structure and diameter, as well as exemplary material which may be used to prepare particular NP) depending on the nature of the raw material from which these structures can be obtained.

Figure 1 shows a schematic structure of the described NPs. Available data from animal models demonstrated that, regardless of the route of administration of a drug, they tend to penetrate into the bloodstream quickly and are then distributed in organs and tissues. Their elimination from the body is slow. Most tissues and organs are able to remove NPs after cessation of exposure (with the exception of the reproductive organs and brain, which have been found to have increased retention). Nanoparticles can be eliminated from the body by the mononuclear phagocyte system (MPS) and by organs with the extensive reticuloendothelial system (RES) such as the liver and spleen. ${ }^{32}$ In turn, nanocarriers based on biodegradable polymers (e.g., lactic acid polymers (PLA), glycol acid polymers (PGA) or a mixture of them (PLGA)) are completely degraded in the body, and their degradation products are excreted in the urine after reaching the renal threshold. ${ }^{33}$ 


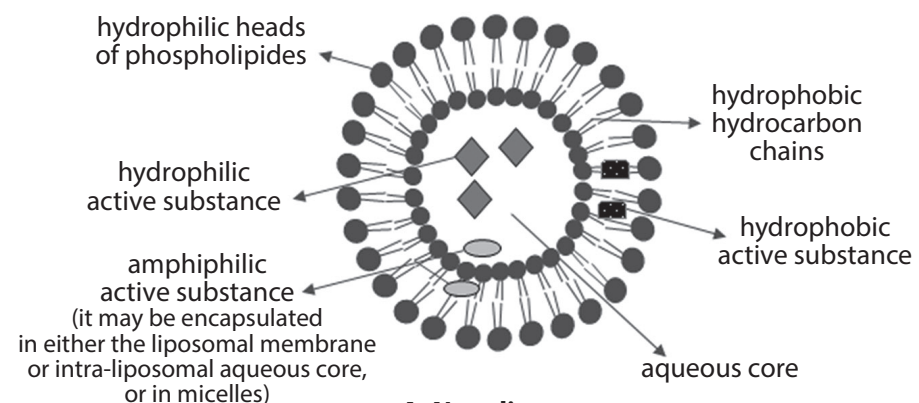

or in micelles) $\quad$ A. Nanoliposome
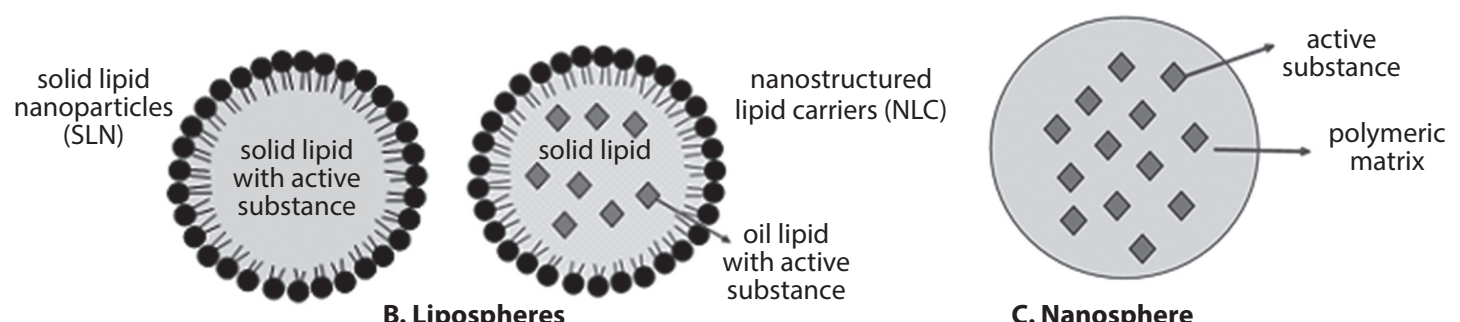

C. Nanosphere

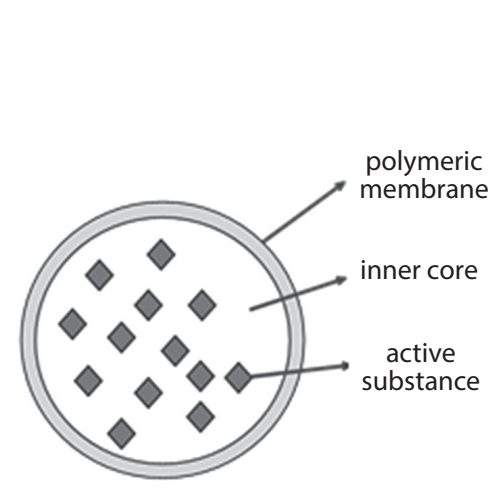

D. Nanocapsule

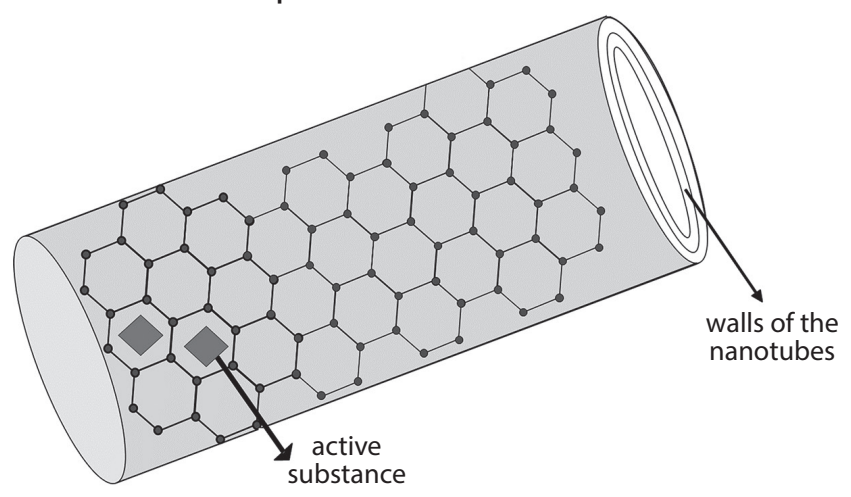

F. Carbon nanotube

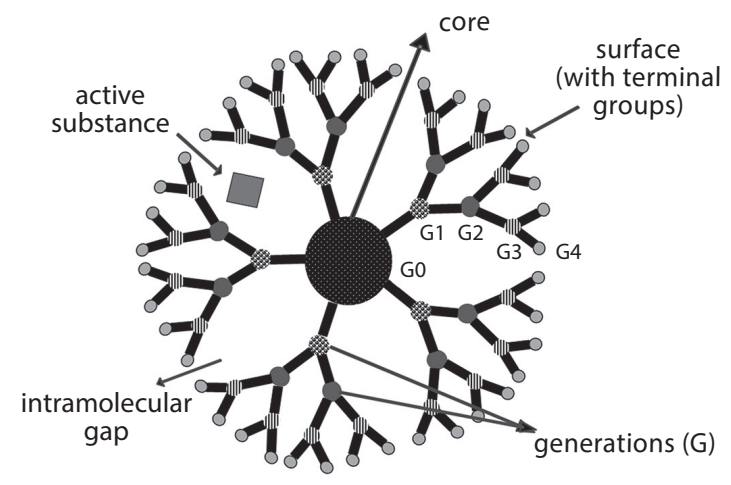

E. Dendrimer

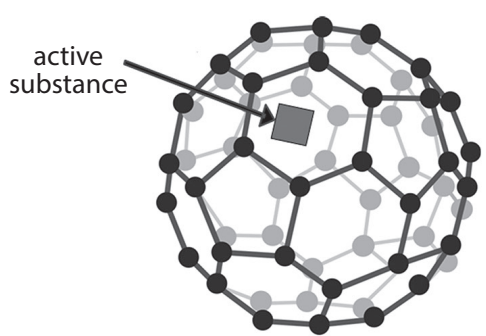

G. Fullerene

Fig. 1. Schematic structure of selected nanocarriers of active substances: (A) nanoliposome; (B) lipospheres; (C) nanosphere; (D) nanocapsule; (E) dendrimer; (F) carbon nanotube; (G) fullerene (original drawings)

The toxicity of NPs correlates with their physicochemical properties, i.e., size, shape, chemical composition, surface structure, surface charge, degree of aggregation, solubility, degradation time, presence or absence of functional groups of other chemical compounds, as well as reactivity with the surrounding tissue. Nanoparticles may affect the phagocyte system by initiating stress reactions, and consequently inflammation. Slowly decomposing and/or non-biodegradable NPs may accumulate in internal organs. They are able to pass through cell membranes, while inhaled NPs may reach the liver and heart via the bloodstream. Nanoparticles may affect the proper course of biochemical processes in the human body, including regulatory mechanisms of enzymes and proteins. Table 2 summarizes the possible adverse effects, which apply to polymer and carbon NPs. 
Table 1. Characteristics of selected NPS

\begin{tabular}{|c|c|c|c|c|c|c|}
\hline $\begin{array}{l}\text { NPs depending } \\
\text { on the nature } \\
\text { of the raw material }\end{array}$ & \multicolumn{2}{|c|}{ Types of NPs } & $\begin{array}{l}\text { Short description } \\
\text { of the particular NP }\end{array}$ & Diameter of NPs & $\begin{array}{l}\text { Exemplary material used for } \\
\text { NPs manufacture }\end{array}$ & Reference \\
\hline \multirow{3}{*}{ Lipid-based NPs } & \multicolumn{2}{|c|}{ nanoliposomes } & $\begin{array}{l}\text { spherical structures with the walls } \\
\text { formed by a lipid bilayer and } \\
\text { an aqueous phase inside }\end{array}$ & $\begin{array}{l}\text { usually below } \\
100-200 \mathrm{~nm}\end{array}$ & $\begin{array}{c}\text { phospholipid, cholesterol } \\
\text { and phosphatidic acid which } \\
\text { contains specified charge, } \\
\text { soybean lecithin }\end{array}$ & $6,11,38$ \\
\hline & \multirow{2}{*}{ lipospheres } & SLN & $\begin{array}{l}\text { structure of SLN resembles the o/w } \\
\text { emulsion; liquid lipid is replaced } \\
\text { by a lipid solid in room temperature }\end{array}$ & \multirow{2}{*}{$\begin{array}{l}\text { lipospheres show } \\
\text { an average size } \\
\text { below } 500 \mathrm{~nm} \text {, } \\
\text { whereby SLN } \\
\text { reach larger sizes } \\
\text { than NLC }\end{array}$} & $\begin{array}{l}\text { glyceryl monostearate } \\
\text { soybean lecithin }\end{array}$ & $6,11,21$ \\
\hline & & NLC & $\begin{array}{l}\text { NLC consist of oil droplets containing } \\
\text { the drug, placed in the solid lipid matrix }\end{array}$ & & $\begin{array}{l}\text { solid and liquid lipids (e.g., } \\
\text { Miglyol, Capmul, MCM) }\end{array}$ & $6,11,21$ \\
\hline \multirow{3}{*}{ Polymer-based NPs } & \multicolumn{2}{|c|}{ nanospheres } & $\begin{array}{l}\text { API is incorporated (suspended) } \\
\text { in a biodegradable polymer matrix }\end{array}$ & $\begin{array}{l}\text { usually } \\
10-200 \mathrm{~nm}\end{array}$ & $\begin{array}{l}\text { PLA, PGA or a mixture of them } \\
\text { (PLGA), PMMA }\end{array}$ & $6-11,35$ \\
\hline & \multicolumn{2}{|c|}{ nanocapsules } & $\begin{array}{l}\text { the form of colloidal nanovesicles } \\
\text { in which the core of this structure } \\
(\mathrm{o} / \mathrm{w}) \text { is surrounded by a polymer } \\
\text { membrane }\end{array}$ & $100-500 \mathrm{~nm}$ & $\begin{array}{c}\text { synthetic (e.g., } \\
\text { polycaprolactone, } \\
\text { polyacrylamide, methyl } \\
\text { polymethacrylate) and natural } \\
\text { (e.g., gelatine, chitosan, } \\
\text { albumins) polymers }\end{array}$ & 6,22 \\
\hline & \multicolumn{2}{|c|}{ dendrimers } & $\begin{array}{l}\text { tree-like structure consisting } \\
\text { of a core (or focal group), the interior } \\
\text { of the polymer and a surface }\end{array}$ & approx. 20 nm & $\begin{array}{l}\text { polylysine and } \\
\text { polyamidoamine, } \\
\text { poly(aryl ether) }\end{array}$ & $\begin{array}{c}6,8,9,23 \\
24\end{array}$ \\
\hline \multirow[t]{2}{*}{ Carbon-based NPs } & \multicolumn{2}{|c|}{ nanotubes } & $\begin{array}{l}\text { cylindrical graphene films which } \\
\text { are open or closed on one or two } \\
\text { sides, with carbon atoms arranged } \\
\text { in hexagonal structures }\end{array}$ & below $25 \mathrm{~nm}$ & their surface can be modified & $\begin{array}{l}6,8,11 \\
26-28\end{array}$ \\
\hline & \multicolumn{2}{|c|}{ fullerenes } & an allotropic form of pure carbon & approx. $1 \mathrm{~nm}$ & $\begin{array}{l}\text { their structure can be modified } \\
\text { by coating with PVP or PEG }\end{array}$ & $\begin{array}{l}6,8,9,11 \\
25,26\end{array}$ \\
\hline
\end{tabular}

NPs - nanoparticles; SLN - solid lipid NPs; NLC - nanostructured lipid carriers; API - active pharmaceutical ingredient; PLA - lactic acid polymers; PGA - glycol acid polymers; PMMA - poly(methyl methacrylate); PVP - polyvinylpyrrolidone; PEG - polyethylene glycol.

Table 2. Possible adverse effects of selected NPs

\begin{tabular}{|c|c|c|}
\hline $\begin{array}{l}\text { NPs depending } \\
\text { on the nature } \\
\text { of the raw material }\end{array}$ & $\begin{array}{l}\text { Types } \\
\text { of NPS }\end{array}$ & Possible adverse effects \\
\hline \multirow[b]{2}{*}{ Polymer-based NPs } & nanospheres & $\begin{array}{l}\text { Cytotoxicity depends on size, shape and surface charge. } \\
\text { The small size of the nanospheres and the aggregation process are the main causes for their cytotoxicity. }\end{array}$ \\
\hline & dendrimers & $\begin{array}{l}\text { Dendrimer cytotoxicity depends on the generation, the number of surface groups, and the nature of terminal } \\
\text { moieties (anionic, neutral or cationic). Higher-generation dendrimers as well as dendrimers with positive charges } \\
\text { on the surface present higher cytotoxicity. }\end{array}$ \\
\hline \multirow{2}{*}{ Carbon-based NPs } & nanotubes & $\begin{array}{l}\text { Similarly to asbestos fibers, carbon nanofibers are likely to induce some pro-inflammatory mechanisms, i.e., the secretion } \\
\text { of IL-1 } \beta \text { from LPS-primed macrophages. In addition, CNT may cause genotoxic effects. }\end{array}$ \\
\hline & fullerenes & $\begin{array}{l}\text { Fullerene colloid prepared with tetrahydrofuran demonstrates toxic effects in different mammalian cells, including } \\
\text { mitochondrial depolarization, and lipid peroxidation resulting in necrotic cell death. }\end{array}$ \\
\hline
\end{tabular}

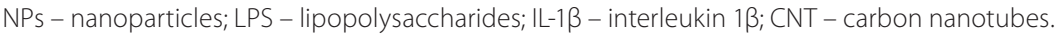

\section{Application of drug nanocarriers in selected diseases}

The use of NPs in medicine and pharmacy was mentioned earlier as being intensively studied in the diagnosis and treatment of a rising number of diseases. In this section, we discussed the results of studies analyzing the use of API nanocarriers in selected chronic diseases, i.e., cardiovascular disease, cancer and neurodegenerative diseases, as well as in wound healing. Since this topic is so broad, we have limited our discussion to results which we believed are the most interesting, relevant or innovative.

\section{The potential use of nanoparticles in cardiovascular disorders}

For several years, research on the possibilities of using nanotherapy to treat embolism and thrombosis has been conducted. ${ }^{34}$ Nanoparticles loaded with fibrinolytic drugs 
have been analyzed. They work to dissolve blood clots, a cause of myocardial infarctions, strokes and pulmonary embolisms. Korin et al. developed a targeted DDS to eliminate blood clots. ${ }^{35}$ Nanoparticles were obtained using spray-drying solutions containing poly(lactic-co-glycolic acid) (PLGA 50:50, MW $17 \mathrm{kD}$ ). The surface of the NPs was coated with a tissue plasminogen activator (t-PA). Tissue plasminogen activator is bound by surface conjugation with the use of streptavidin/biotin. The authors tested the nanodrug activated by shear stress in a model of pulmonary embolism and mesenteric artery embolism in mice. From a carrier sized $180 \pm 70 \mathrm{~nm}$ they obtained microaggregates of a size similar to platelets, which were stable under physiological blood flow conditions. Within the artery narrowing, where the blood flow increases rapidly (shear stress of $1,000 \mathrm{dyn} / \mathrm{cm}^{2}$ ), there was a spontaneous activation of the nanodrug, i.e., the breakdown of the microaggregate into NPs with t-PA. Korin et al. found that the released NPs accumulated within the thrombus and exhibited pharmacological activity. Dissolution of clots was obtained with a therapeutic dose of t-PA of $50 \mathrm{mg}$, which was 50 -fold lower than a standard dose of $\mathrm{t}-\mathrm{PA}$ administered intravenously, i.e., $2 \mathrm{mg} / \mathrm{kg} .{ }^{35}$ However, it is suggested that the NP-t-PA conjugation (biotin and streptavidin) may elicit an immune response. ${ }^{36}$

Lipid-based NPs, i.e., nanoliposomes as well as SLN are among the essential NPs in the targeted therapy for heart diseases. Dvir et al. investigated the efficacy of API encapsulation in nanoliposomes in the targeted therapy for myocardial infarctions. Nanoliposomes with a diameter of $142 \mathrm{~nm}$ were conjugated with a ligand specific for the angiotensin II type 1 (AT1) receptor. The obtained lipid-based NPs were administered by intravenous injection to an isolated rat heart and demonstrated high therapeutic efficacy. ${ }^{37}$ Shao et al. investigated the therapeutic effect of Schisandrin B (Sch B) in the form of SLN in the model of the rat myocardial infarction. ${ }^{38}$ Schisandrin B is isolated from the fruit of Schisandra chinensis and can be clinically applied in myocardial ischemia. Polyethylene glycol (PEG) and matrix metalloproteinase-targeting peptide (MMP-TP) conjugate was synthesized in the study. The MMPSch B liposomes, $130 \mathrm{~nm}$ in size, were prepared using the solvent displacement technique. The aqueous phase was prepared by dissolving the PEG-peptide, DOTAP (liposomal transfection reagent) and poloxamer in water. Glyceryl monostearate (GMS), COMPRITOL ${ }^{\circledR} 888$ ATO (Gattefosse, Paramus, USA), which can serve as a lipid carrier, and soybean lecithin (SL), were sonicated in acetone to form an organic phase. Both phases were combined and mixed until the organic solvent was removed. Efficacy and biodistribution of the obtained nanodrug were tested both in vitro and in vivo. The authors demonstrated that the developed formulation is the optimal carrier of API for the myocardium tissues and effectively protects the heart against acute myocardial infarction. ${ }^{38}$

The possibility of using porous silicon nanoparticles (PSi) in cardiovascular disease therapy was also assessed. The Finnish team attempted to develop PSi-based nanosystems for targeted therapy and diagnosis of myocardial infarction. Peptide-modified nanovectors PSi NPs sized $10.7 \mathrm{~nm}$, with a surface area of $282 \mathrm{~m}^{2} / \mathrm{g}$ and a total pore volume of $0.75 \mathrm{~cm}^{3} / \mathrm{g}$, were manufactured in the course of the study and analyzed in terms of their distribution in the myocardium tissue after intravenous administration in the rat model. ${ }^{39}$ The results obtained by the cited authors are promising and indicate the potential application of this formulation in the future. Similarly, positive results were demonstrated for thermally oxidized porous silicon (TOPSi) NPs in the isolated rat heart model. ${ }^{40}$ The NPs presented biocompatibility and did not significantly affect cardiac function both before and after the myocardial infarction. In turn, Chan et al. conducted research on the safety of using silicon nanoparticles (SiNPs). ${ }^{41}$ The toxicity of SiNPs, which were $150 \mathrm{~nm}$ in size, was measured in vivo in a mouse model. The mice were administered NPs intravenously and underwent observation for 14 days. After this time, the authors performed histopathological examinations and blood analysis to assess internal organ damage. In this study, the occurrence of adverse effects after the administration of SiNPs was not confirmed. ${ }^{41}$ However, some studies have suggested that intravenous administration of SiNPs may lead to their distribution/accumulation in the liver, spleen and lungs, with subsequent damage of these organs. ${ }^{42}$

Myocardial infarction can lead to organ failure, thereby necessitating transplantation. Unfortunately, the number of people waiting for a heart transplant exceeds the number of available grafts; therefore, stem cell therapy seems prospective. ${ }^{43}$ However, their survival is limited due to oxidative stress and ischemia-induced inflammation. Ma et al. suggested the usage of melatonin, which is a powerful endogenous antioxidant, for protecting cells against oxidative stress. ${ }^{44}$ In order to increase the effectiveness of this antioxidant, the authors developed a technique for melatonin encapsulation in PLGA-mPEG nanoparticles (Mel-NPs). They observed that Mel-NPs achieved a higher melatonin bioactivity index over time and a better therapeutic effect than a conventional melatonin preparation in the model of rat myocardial infarction. ${ }^{44}$ Myocardial infarction therapy using PLGA NPs was also proposed by Nakano et al. ${ }^{45}$ who developed bioabsorbable irbesartan-loaded PLGA NPs. The efficacy of irbesartan-NPs was studied in the model of ischemia-reperfusion injury in the mouse heart, showing that such NPs may affect the limitation of infarct size. ${ }^{45}$

Promising results were also shown in the case of the therapeutic use of proteins in myocardium regeneration after myocardial infarction. A major barrier to the success of this therapy is the short half-life of proteins in vivo. It has been found that liraglutide loaded in PLGA-PEG 
NPs (liraglutide-NPs) provides sustained release of API and retains its biological activity in vitro. ${ }^{46}$ In addition, in the model of rat myocardial infarction, liraglutide-NPs improved heart function, reduced the extent of myocardial necrosis, promoted angiogenesis, and also prevented the apoptosis of cardiomyocytes. ${ }^{46}$ Zhang et al. have shown that the cytotoxicity of PLGA-PEG NPs depends on their shape. ${ }^{47}$ Needle-shaped NPs induced cytotoxicity in the tested cell lines. Lysosomal membrane damage, lysosomal enlargement, caspase-3 activation, and DNA damage were observed, which in turn induced cell apoptosis. The authors did not observe this process in the case of spherical PLGA-PEG NPs. ${ }^{47}$

Currently, attempts are being made to develop inhalation therapy, which would use biocompatible and biodegradable peptide NPs to treat heart failure. ${ }^{48}$ The therapeutic efficacy of peptides delivered in a calcium phosphate nanoparticles (CaP-NP) with a size $<50 \mathrm{~nm}$ in the rat and mouse models of diabetic cardiomyopathy was confirmed. ${ }^{48}$ The proposed carrier allows for rapid distribution of the peptide from the lungs to the bloodstream, and subsequently to myocardium and cardiomyocytes. This study revealed that heart function was regenerated and improved.

\section{The potential use of nanoparticles in the treatment of cancer}

Cancer is a complex disease and still very difficult to treat. Classic radiotherapy, as well as chemotherapy, is not very effective, mainly due to the lack of specificity, low bioavailability and drug resistance. ${ }^{49}$ Nanoscale technology may be a promising tool to overcome the limitations of conventional anticancer therapy. Because the NPs are coated with specific ligands or antibodies, an increased concentration of the drug in the tumor was found, along with reduced cytotoxicity in relation to healthy cells. The reason for this is that the ligand binds specifically to receptors on the surface of tumor cells, often those that overexpress the disease-related ones. In breast cancer, overexpression of the human epidermal growth factor 2 (HER2) is often observed..$^{50}$ The recombinant, humanized monoclonal antibody - trastuzumab - is used against the HER2 protein. It has been proven that cancer cells with an increased expression of the HER2 receptor show a more frequent and more intense drug resistance. ${ }^{51}$ However, despite the potential for greater malignancy, their response to the combined treatment is very effective. Nonetheless, cytotoxicity of healthy cells remains an important problem.

In the case of polymeric-based NPs, many reports considered the use of dendrimers, especially polyamidoamine (PAMAM) dendrimers, ${ }^{52}$ which come from polyamidoamine. Their core may be composed of a hydrophilic ethylenediamine ${ }^{24}$ or, for example, of a hydrophobic diaminododecane. Kulhari et al. synthesized dendrimers with trastuzumab to facilitate the delivery of docetaxel to breast cancer cells. ${ }^{53}$ The use of such a conjugate resulted in positive effects related to the possibility of reducing the dose of cytostatics, thus reducing the cytotoxicity in relation to healthy cells, but also reducing drug resistance. ${ }^{53}$ The same type of dendrimer, but doxorubicin-loaded, was tested in mice by Zhong et al. ${ }^{54}$ to analyze the possibility of reducing the lung metastases. Decreased cardiotoxicity was found in addition to increased accumulation of the drug in the tumor.

Unfortunately, apart from such promising reports related to the use of dendrimer conjugates with anticancer drugs, some rather more negative aspects of such therapies should be mentioned. All types of dendrimers are characterized by cytotoxic and hemolytic properties. ${ }^{24}$ To eliminate or reduce these disadvantages, the surface of dendrimers can be modified, e.g., by binding to PEG (PEGylation). PEGylation, in addition to reducing cytotoxicity, carries another positive feature: it reduces the release of the drug from the formulation. ${ }^{55}$ Furthermore, it was found that PEGylation did not affect the load capacity of the drug. ${ }^{55}$

Recently, the use of Au-Ag NPs in bladder cancer has also been analyzed. ${ }^{56}$ Polydopamine (PDA)-coated Au-Ag molecules (Au-Ag@PDA NPs) were administered to T24 cells at different doses. These cancer cells were then irradiated with a laser wavelength of $808 \mathrm{~nm}$. In addition to the in vitro study, the authors also verified the effects of therapy with the use of Au-Ag@PDA NPs in mice. Good structural stability, biocompatibility, photothermal effects, and potential anticancer efficacy were found. ${ }^{56}$

Similarly to dendrimers, carbon NPs can be conjugated to a ligand specific for a particular receptor and overexpressed in a given cancer. Among carbon-based NPs which can be used in cancer therapy, special attention has been paid to CNT and carbon dots (C-dots). These NPs were suggested as carriers of doxorubicin and gemcitabine. An interesting perspective is the coupling of CNT with cadmium telluride quantum dots (CdTe-QDs). This allows for optical imaging and drug administration using an external magnetic field. The CNT were filled with $\mathrm{Fe}_{3} \mathrm{O}_{4}$. On their surface, quantum dots coated with silica $\left(\mathrm{SiO}_{2}\right)$ were added. Magnetite crystals improved chemical stability, increased the load capacity of the drug and protected against agglomeration. Silica hybrid coatings reduced the toxicity of the entire system. The excellent load capacity of this nanosystem was discovered together with the possibility of delivering directional doxorubicin hydrochloride to Hela cells. ${ }^{57}$

On the other hand, in the study by Shi et al., ${ }^{58}$ the outer part of MWCNT was conjugated with PAMAM dendrimers. Such a nanosystem was observed to have high stability and biocompatibility. It was also effective in reaching cancer cells overexpressing folic acid receptors with high affinity, which can undoubtedly be used both in cancer diagnostics and therapy. 
In the case of fullerenes, the data indicated that anticancer drugs conjugated with fullerenes may decrease their cytotoxicity on account of their role as radical sponges. ${ }^{38,39}$ In the study of Chaudhuri et al., ${ }^{59}$ conjugate of fullerenoldoxorubicin (DOX) inhibited the proliferation of cancer cells in vitro, blocked the G2-M cell cycle and induced the process of apoptosis. The authors did not observe any toxicity of the obtained nanosystem, which even demonstrated its protective effect on cardiac muscle, kidney, lung, as well as venereal cells. Similar cardioprotective results were demonstrated in the study performed on Wistar rats by Torres et al. ${ }^{60}$

Forms and structures of different types of NPs allow for their usage in thermal and photodynamic therapy. ${ }^{61}$ Recently, it has been found that phototherapy, induced utilizing a near-infrared laser with the use of modern photosensitizers, brings better treatment effects than previously used therapies. In their study, Sheng et al. used copper oxide sulfide NPs with polyethylene glycol diacrylate modified with copper sulfide (CuS) NPs (PEG-DA-CuS NPs) ${ }^{61}$ Due to PEGylation, the solubility of $\mathrm{CuS}$ was increased, with a subsequent increase of its bioavailability. When these NPs reached the tumor, they were irradiated with a laser, which increased their temperature above $90^{\circ} \mathrm{C}$.

For lipid-based NPs in the treatment of cancer, Yang et al. used lipospheres SLN, obtained using high-pressure homogenization, as carriers of the anticancer drug - camptothecin. ${ }^{62}$ The authors have shown that SLN may prolong the release of camptothecin and other lipophilic drugs for even 1 week. In other research, linalool, which is a monoterpene found in essential oils of plants and herbs showing an antiproliferative activity in cancer cells, was encapsulated in SLN lipospheres with a yield of over $80 \% .{ }^{63}$ Controlled in vitro release profiles over a period of at least $72 \mathrm{~h}$ were obtained for this nanosystem. The authors also demonstrated the antiproliferative effects of SLN-loaded linalool on hepatocarcinoma (HepG2) and lung adenocarcinoma (A549) cells as well as higher inhibitory effects compared to free linalool.

Zhou et al. ${ }^{64}$ encapsulated quercetin (QCT) into nanoliposomes and evaluated their morphology, particle size distribution, drug loading, encapsulation ratio, and the in vitro release of the drug. In addition, the authors carried out pharmacokinetics and anticancer activity examination on the mice model. Nanoliposomes were prepared using the thin-film hydration method with a modification using a short peptide containing arginine-glycine-aspartic acid (RGD). It was demonstrated that water-insoluble reagents, such as QCT, are readily dispersed in lipid solution and entrapped by the thin-film hydration. In addition, it was found that the size of the obtained nanoliposomes is suitable for the fenestrated blood vessels of the cancer tissues through the enhanced permeability retention effect. The biodistribution was evaluated using in vivo fluorescence imaging giving strong signals around the carcinoma cells. The release tests showed that the proposed QCT delivery system is useful because of its accumulation in cancer tissues and the reduced exposure of healthy tissues. The use of the RGD peptide to target integrin in the endothelium of the blood vessels of the tumor has confirmed the feasibility of a previously known method to inhibit angiogenesis and metastasis. The results indicate that the proposed RGD complex with nanoliposomes significantly increases the ability to inhibit tumor growth compared to normal liposomes. ${ }^{64}$

In the study conducted by Hasan et al., ${ }^{65}$ nanoliposomes containing curcumin, which is characterized by a broad spectrum of positive activities, e.g., antioxidant, antitumor, anti-inflammatory, as well as antimicrobial, were prepared using lecithin from salmon, soya and rapeseed. The average size of the obtained nanoliposomes was the smallest for those made from soya $(110.3 \pm 0.8 \mathrm{~nm})$ when compared with free liposomes and rapeseed and salmon nanoliposomes. The authors observed the highest entrapment efficiency of the curcumin in the case of salmon liposomes $(67.3 \% \pm 1.1 \%$ vs $63.2 \% \pm 0.7 \%$ for rapeseed nanoliposomes and $65.0 \% \pm 1.1 \%$ for soya nanoliposomes). The in vitro antitumor activity of liposomal curcumin was analyzed on MCF7 cancer cells. The study demonstrated that curcumin-loaded nanoliposomes significantly increased the cancer cell cytotoxicity, while lower impact of free curcumin on cancer cells was found.

In turn, Chen and $\mathrm{Liu}^{66}$ received nanoliposomes with bufalin (BF) using the high-pressure homogenization method. Empty liposomes (LP), liposomes modified with folic acid (FA-BF-LP), liposomes modified with transferrin (Tf-BF-LP), and complex liposomes modified with both FA and Tf (FA+Tf) BF-LP were administered into the mice with lung cancer. Bufalin has antiproliferative properties and induces apoptosis of tumor cells. However, it is toxic, insoluble in water and has a short half-life. Due to the abovementioned features, the authors focused on the possibility of closing BF into modern DDSs. They determined the morphology of the NPs as being spherical and of appropriate size. Particle size is an important parameter. Because NPs below $400 \mathrm{~nm}$ can emerge in the microspace of the tumor, smaller sizes can internalize into the cell through endocytic vesicles in a more efficient way. The anticancer efficacy was assessed after a period of 2 weeks after injection into A549 cells. The greatest effect of tumor suppression was observed after the (FA+Tf) BF-LP administration. ${ }^{66}$

In a study by Zabielska-Koczywąs et al., ${ }^{67}$ gold NPs (Au NPs) loaded with doxorubicin (DOX) and glutathione-stabilized (GSH) were analyzed in nude mice in feline injection-site sarcomas, which are malignant skin tumors. The obtained Au-GSH NPs had a size of approx. $5.5 \mathrm{~nm}$. The Au-GSH NPs were observed to co-internalize with tumor-associated macrophages (TAM) close to the area of necrosis and in the tumor periphery. Further, the authors found no negative effect of AU NPs on liver and kidney parameters in nude mice. ${ }^{67}$ 


\section{The potential use of nanoparticles in delivering API to the brain}

The most important problems in the development of new drugs for these diseases are ineffective crossing of bloodbrain barrier (BBB), the poor solubility of the drug as well as its low bioavailability. The size of active substance particles and endothelial permeability are the main factors that affect the crossing of the $\mathrm{BBB}$, which separates the central nervous system (CNS) from the systemic circulation. Among other barriers limiting or preventing drug delivery to the brain, a barrier separating the blood from the cerebrospinal fluid (blood-cerebrospinal fluid barrier (BCSFB)) and some functional barriers in the forming transporter mechanisms (influx and efflux) of the CNS may be distinguished. ${ }^{68}$

A number of studies have been performed to analyze whether nanotechnology methods allow the delivery of active substances to the brain. ${ }^{69-73}$ Experimental research on animal models demonstrated the effective delivery of nanoemulsions containing diazepam and risperidone into a rat brain. ${ }^{69,70}$ Nanoemulsion with diazepam was prepared with the use of cold high-pressure homogenization and the following ingredients: triglycerides and soybean oil (oil phase), 0.1 M phosphate-buffered saline (PBS; pH 8, aqueous phase), and lecithin and polysorbate 80 (emulsifiers). Fast and intense initial distribution of diazepam into rat brain was demonstrated when nanoemulsions with $20 \%$ and $30 \%(\mathrm{w} / \mathrm{w})$ oil content were used. ${ }^{69}$ Another study of the same research team compared the effectiveness of different emulsifiers used during the preparation of nanoemulsions and showed that risperidone brain availability was increased in the case of poloxamer 80 in comparison with that of poloxamer 188 or Solutol ${ }^{\circledR}$ HS15 (Sigma-Aldrich, St. Louis, USA). ${ }^{70}$

Sadegh Malvajerd et al. ${ }^{71}$ demonstrated that after the intravenous administration of a $4 \mathrm{mg} / \mathrm{kg}$ dose of curcumin in a rat, its amount available in the brain was significantly higher in the case of curcumin-loaded nanostructured lipid carriers than in the case of free curcumin and curcumin-loaded SLN. Curcumin was dispersed as amorphous in the analyzed nanocarriers.

Recently, some interesting studies showing the possible delivery of active substances to the brain via the nasal route have been published. ${ }^{72,73}$ Liu and $\mathrm{Ho}^{72}$ manufactured chitosan NPs using an ionic cross-linking method, loaded them with scutellarin (SCU), which is a traditional Chinese medicine used for the treatment of ischemic cerebrovascular disease, and aimed to deliver SCU to the brain through the nasal route. Increased accumulation of SCU delivered as encapsulated in NPs in the brain was observed when compared to SCU in solution. In a study by Sharma et al., ${ }^{73}$ PLGA midazolam-loaded NPs delivered intranasally demonstrated a drug release at $83 \%$ within $4 \mathrm{~h}$ and can, therefore, be proposed as a non-invasive DDS which improves drug entrapment and stability.

\section{Neurodegenerative diseases}

Since the number of people suffering from neurodegenerative diseases is increasing each year, they are a serious health problem all over the world. ${ }^{74}$ The course of dementia, especially Alzheimer's disease (AD), has most often a slow beginning and may remain unnoticed for a long time. However, their impact on patients as well as the families' lives is enormous, both during the development of the disease and after the diagnosis. ${ }^{74}$ Unfortunately, so far no drug has been developed to completely counteract the effects of neurodegeneration. Thus, the only way to reduce its progression is through early diagnosis and subsequent successful treatment.

Alzheimer's disease is caused by toxic effects of $\beta$-amyloid peptides that aggregate and are deposited as amyloid plaques in neural and vascular tissues. It all begins with the proteolytic cleavage of the amyloid precursor protein (APP) ${ }^{75}$ It has also been shown in animal models that dysfunction of the cholinergic system can produce a memory deficit similar to AD.$^{76}$ Current treatment of AD is based on acetylcholinesterase (AChE) inhibitors or influencing inhibition of glutamate excitotoxicity. ${ }^{77}$ However, tacrine, galantamine, rivastigmine, and memantine have been linked to some adverse effects including hepatotoxicity, nausea, vomiting, dizziness, confusion, or constipation, which may lead to the discontinuation of the treatment. ${ }^{77}$ In the brains of rats, Wilson et al. ${ }^{78}$ observed that the level of tacrine, which was encapsulated in NPS coated with $1 \%$ nonionic surfactant polysorbate 80 , was elevated compared with the uncoated NPs as well as with the free drug. According to the authors, these coated NPs may be delivered to the brain through the interaction between polysorbate 80 coating and the endothelial cells of the brain microvessels. ${ }^{78}$ What is more important, the toxicity of tacrine may be reduced with this drug form, as coating tacrine-loaded NPs with polysorbate 80 decreased its accumulation in the liver and spleen. Other studies also demonstrated the role of polysorbate 80 in the effective delivery of different active substances into the brain. ${ }^{79,80}$

Because of their many advantageous effects, nanoliposomes have been suggested to be a promising system for API delivery to the brain in AD, since they are non-toxic, biodegradable and non-immunogenic. The study conducted by Truran et al. ${ }^{81}$ demonstrated that nanoliposomes composed of phosphatidylcholine, cholesterol and phosphatidic acid with a 70:25:5 molar ratios (20 mg lipid/mL) prevent $\beta$-amyloid peptide 1-42 (A $\beta 42$ ) fibril formation. Nanoliposomes were prepared through the dissolution of the lipid mixture in chloroform, which was subsequently removed using a rotary evaporator, and then the dry lipid film was hydrated using a HEPES buffer and the mixture was sonicated. ${ }^{81}$ A curcumin-decorated nanoliposomes was demonstrated to have a very high affinity for A $\beta 1-42$ fibrils with their potential in the targeted delivery of new 
diagnostic and therapeutic molecules for AD.$^{82}$ Very promising results have been linked to the studies concerning dendrimers as carriers of anti-amyloidogenic and anti-prionic substances. ${ }^{83,84}$ The study conducted by Klementieva et al. ${ }^{83}$ demonstrated that in vitro poly(propylene imine) (PPI) glycodendrimers of the $4^{\text {th }}$ and $5^{\text {th }}$ generation modified with maltose can reduce the toxicity of $A \beta(1-42)$. Furthermore, Klajnert et al.$^{84}$ found a reduction of $\beta$-amyloid fibril formation when using gallic acid-triethylene glycol (GATG) dendrimer decorated with 27 terminal morpholine groups ([G3]-Mor).

In the case of using carbon-based NPs in the AD model, the study of Li et al. ${ }^{85}$ demonstrated that SWCNT had the potential to inhibit $A \beta(16-22)$ and full-length $A \beta$ fibrillation. The study conducted by Yang et al. ${ }^{86}$ demonstrated the successful delivery of acetylcholine to mice brains with experimentally induced AD using SWCNT with a diameter of $0.8-1.2 \mathrm{~nm}$ and a length of several microns. The authors precisely controlled the doses of obtained acetylcholineloaded SWCNT, ensuring that the SWCNT preferentially penetrates the lysosomes instead of the mitochondria, which can happen when using high doses. In turn, Lohan et al. ${ }^{87}$ indicated the potential role of MWCNT, coated with polysorbate/phospholipid and berberine-loaded, in reducing $\beta$-amyloid-induced AD. The analyzed formulation had a size of $186 \mathrm{~nm}$ and manifested $96 \%$ release of berberine over $16 \mathrm{~h}$.

In Parkinson's disease (PD), overexpression of $\alpha$-synuclein leads to the death of dopaminergic neurons. Therapy is limited to relieving the symptoms of the disease, both by using therapeutics or surgery. ${ }^{88}$ At present, there is no treatment which could lead to regeneration of the brain tissue. However, many different neuroprotective and neuroregenerative molecules, including neurotrophic factors, antioxidants and RNA-based drugs, have been studied for their therapeutic capabilities in PD. ${ }^{89}$ Unfortunately, their delivery to the brain is still a big challenge. Recently, a therapeutic device, which has the potential to deliver cerebral dopamine neurotrophic factor directly to the brain of PD patients, has also been designed..$^{90}$ There are also many reports indicating the advantages of using NPs in PD therapy. ${ }^{10}$

Hu et al. ${ }^{91}$ analyzed Au NPs loaded with plasmid DNA and obtained through the combination of electrostatic adsorption and photochemical immobilization methods with regard to their therapeutic effects in PD models. ${ }^{91}$ The authors observed that these systems could successfully cross the BBB and inhibit the apoptosis of PC12 cells and dopaminergic neurons in PD mice brain. In a study conducted by Cao et al., ${ }^{92}$ rat models of PD were separately given chitosan-coated levodopa liposomes/benserazide and levodopa/benserazide. The authors found that levodopa-loaded liposomes, coated with chitosan, may reduce dyskinesias inducing PD. In another study concerning the usage of nanoliposomes in the PD model, neutral (zwitterionic) nanoliposomes supplemented with cholesterol
(NLP-Chol), both PEGylated or not, were demonstrated to reduce the neurotoxicity of $\alpha$-synuclein, which aggregates to form fibrils in neuronal cells of PD patients. ${ }^{93}$

Interesting results were demonstrated by Al-Dhubiab et al. ${ }^{94}$ who prepared selegiline-loaded poly(lactide-coglycolide) nanospheres, which then served to impregnate a buccal film made with hydroxypropylmethylcellulose and eudragit. The physical properties, mucoadhesive strength and hydration of the film were found to be adequate. In vivo analysis of the buccal film revealed improved bioavailability in comparison with the oral solution, making it a possible alternative approach for the treatment of PD. ${ }^{94}$

Inhibiting the $\alpha$-synuclein fibril formation is a potential therapeutic strategy in PD. Studies analyzing the possible role of carbosilane and viologen-phosphorus dendrimers in this process have previously been performed. ${ }^{95,96}$ The authors also demonstrated carbosilane dendrimers as a partially protective factor against the toxic effect of rotenone, which is a compound of pesticides. ${ }^{96}$ Rotenon is suggested to be a risk factor for PD, since it induces oxidative stress, aggregation of $\alpha$-synuclein and dysfunction of the ubiquitin-proteasome system.

\section{The potential use of nanoparticles in the treatment of wounds}

Chronic wounds have a significant impact not only on patients' life, resulting in impaired mobility, limb amputation and even death, but also on the healthcare system. Wound healing is a complicated process that needs immediate management after the onset of injury. Ideal wound healing should be rapid, leaving minimal scarring with no negative esthetic effects. Many stages are involved in the whole process of healing, including coagulation, inflammation at the sites, proliferation, angiogenesis, remodeling, and restructuring of the tissues. The exact mechanisms of wound healing are not fully understood; however, it has been suggested that the use of silver NPs (Ag NPs) on wounds may cause a reduction in the activity of local matrix metalloproteinases and increase the destruction of neutrophils in the wound cells. ${ }^{97}$ Silver NPs, which may be an alternative to antibiotics in the treatment of severe open wounds due to the increasing resistance of pathogenic bacteria to antibiotics, are intensively studied. ${ }^{98-100}$ Silver-loaded NPs may reduce the levels of proinflammatory cytokines and, subsequently, accelerate wound healing or inhibit the action of tumor necrosis factor $\alpha$ (TNF- $\alpha$ ) and interferon gamma (IFN $\gamma$ ) factors, which are important in the inflammation process. ${ }^{99}$ However, the issue of silver accumulation and toxicity to the skin should also be considered. Experimental data indicates that absorption of Ag NPs through intact and damaged skin is detectable, while in the case of continuous skin continuity absorption is less pronounced. ${ }^{100}$ The authors also 
observed that in the case of damaged skin, the permeation of silver is higher than in healthy skin.

Ziv-Polat et al. stabilized the thrombin through binding it to maghemite (gamma-Fe(2)O(3)) NPs and compared its wound healing efficiency to free thrombin. ${ }^{101}$ The authors treated incisional wounds on rat skin with the following: a mixture of fibrinogen, $\mathrm{CaCl}(2)$ solution and free or bound thrombin. The highest skin tensile strength after 28 days of therapy was demonstrated for thrombin bound to maghemite NPs and the finding was also confirmed during histological examination. ${ }^{101}$

In turn, Au NPs with epigallocatechin gallate and $\alpha$-lipoic acid significantly accelerated wound healing through their anti-inflammatory and antioxidation effects both in cell culture and a murine model. ${ }^{102}$ In the process of wound healing, Au NPs may also be used to deliver antioxidants as well as nucleic acids. Previous studies have shown the ability of Au NPs, based on antimicrobial peptide combined with pro-angiogenic (vascular endothelial growth factor (VEGF)) plasmids, to promote angiogenesis and inhibit bacterial infection in diabetic wounds, resulting in faster re-epithelization, improved granulation tissue formation and high VEGF expression. ${ }^{103}$ Curcumin NPs were found to have strong antibacterial activity to suppress the growth of Pseudomonas aeruginosa and Staphylococcus aureus. ${ }^{104}$ It was also demonstrated that curcumin NPs could reduce inflammation and induce cell expansion, which is useful in the reconstruction of damaged tissue. ${ }^{105}$

Nanoliposomes containing propylene glycol were previously observed to have elevated flexibility, as well as good stability and biocompatibility, and, therefore, may be a novel topical carrier of active substances. ${ }^{106}$ Kianvash et al. obtained curcumin-propylene glycol liposomes approx. $145 \mathrm{~nm}$ in size to heal second-degree burns in the animal model. ${ }^{107}$ The prepared nanoliposomes, containing $0.3 \%$ curcumin, demonstrated effectiveness with no adverse effects on intact skin. No detectable cytotoxicity on human dermal fibroblast was also found for $0.3 \%$ curcumin-loaded nanoliposomes. In turn, ethosomal $0.2 \%$ curcumin formulation applied once daily on rat dorsal improved the following aspects of wound repair: reepithelization, neovascularization and collagen synthesis, among others. In addition, this formulation significantly inhibited the growth of the burn bacterial flora, including Pseudomonas aeruginosa. ${ }^{108}$

In turn, Li et al. ${ }^{109}$ manufactured flexible nanoliposomes with daptomycin (DAP-FL) for topical delivery and bacteriostatic activity towards skin infections. The authors used lecithin and sodium cholate in a ratio of 17:1 (w/w) while lipid to drug ratio was 14:1 (w/w). The mean size of the obtained DAP-FL was $55.4 \mathrm{~nm}$ and the mean entrapment efficiency was $87.85 \% \pm 2.15 \%$. The study demonstrated rapid and efficient antibacterial activity against Staphylococcus aureus and effective therapeutic levels of DAP were maintained for several hours. ${ }^{109} \mathrm{Xu}$ et al. prepared novel liposomes containing a hydrogel core of silk fibroin (SF) loaded with basic fibroblast growth factor (bFGF). ${ }^{110}$ The obtained liposomes showed high encapsulation efficiency of bFGF and their size was about $100 \mathrm{~nm}$. The authors observed that this specific liposomal system significantly improved the stability of bFGF in wound fluids, while maintaining cell proliferation activity with respect to conventional liposomes containing bFGF. Moreover, the bFGF-loaded liposomes with SF core accelerated wound healing very efficiently and induced regeneration of vascular vessels to an extent beyond that seen in free bFGF or conventional liposomes with bFGF. ${ }^{110}$

Since zinc oxide is a compound composed of many enzymes, it has a great impact on biological functions. However, various factors affect the extent of this impact, including microcrystals morphology, particle size, exposure time, concentration, $\mathrm{pH}$, and biocompatibility. In a study by Kim et al., ${ }^{111}$ zinc oxide NPs were demonstrated to reduce mRNA expression of inflammatory cytokines by inhibiting the activation of nuclear factor kappa B cells. Ali et al., ${ }^{112}$ by using the co-precipitation method, were able to manufacture zinc peroxide NPs $\left(\mathrm{ZnO}_{2}-\mathrm{NPs}\right)$ that were $15-25 \mathrm{~nm}$ in size and with a transition temperature of $211^{\circ} \mathrm{C}$. The obtained NPs presented antimicrobial activity against Pseudomonas aeruginosa and Aspergillus niger strains isolated from burn wound infections. Also, the $\mathrm{ZnO}_{2}$-NPs were found to have anti-elastase, antikeratinase and anti-inflammatory properties, which were promising in wound healing in vivo. ${ }^{112}$

On the other hand, studies concerning PLGA NPs in wound healing have also been performed, since PLGA supplies lactate, which accelerates neovascularization. Chereddy et al. ${ }^{113}$ prepared PLGA NPs with a peptide LL37, which modulates wound healing and angiogenesis, and fights infection. The treatment of wounds using PLGALL37 NPs significantly accelerated healing compared to those treated with PLGA or LL37 alone. The authors observed that PLGA-LL37 NPs improved angiogenesis, upregulated interleukin (IL-6) and VEGF expression, and modulated the inflammatory wound response. During the in vitro experiment, it was also demonstrated that these novel PLGA NPs had no effect on the metabolism and proliferation of keratinocytes. ${ }^{113}$

\section{Conclusions}

One of the main benefits of pharmacotherapy using NPs is the reduction of adverse effects. This is due to the significantly reduced dosage of the active substance compared with the currently available drug forms. In addition, the size of NPs and the possibility of their modification make it possible to design innovative solutions in the field of imaging, diagnostics and therapy.

However, the real threat is the free penetration of NPs into any place inside the body with subsequent accumulation 
in internal organs such as the liver, bone marrow or heart. Opponents of the use of nanotechnology in medicine draw our attention to the dangers that this branch of science may bring, including the risk of cancer. ${ }^{114,115}$ Nanoparticles can be embedded into the DNA, which can result in the degradation of nucleic acid and the formation of free radicals. Some data indicated comparable toxicity between CNT and asbestos fibers. ${ }^{30}$

On the other hand, promising results showing better efficacy of anticancer treatment using NPs may change the long-standing conviction that a diagnosis of cancer is a death sentence. Therefore, it seems that further studies will not be discontinued, since there is an urgent need to reduce the toxicity of anticancer drugs, while simultaneously enhancing their effectiveness. However, decision-makers should, undoubtedly, consider developing standards that would regulate the use of nanomaterials in medicine, with special attention given to their impact on the state of the natural environment.

\section{ORCID iDs}

Beata Sarecka-Hujar (1) https://orcid.org/0000-0003-0002-8591 Anna Banyś (1) https://orcid.org/0000-0002-5815-2678 Aneta Ostróżka-Cieślik (1) https://orcid.org/0000-0002-5179-1370 Radosław Balwierz (1) https://orcid.org/0000-0002-6173-2702 Barbara Dolińska (1) https://orcid.org/0000-0003-3035-7417

\section{References}

1. Schmidt $\mathrm{H}$. Chronic disease prevention and health promotion. In: Barrett DH, Ortmann LH, Dawson A, Saenz C, Reis A, Bolan G, eds. Public Health Ethics: Cases Spanning the Globe. Cham, Switzerland: Springer; 2016:137-176.

2. Gasztych M, Komsa K, Musia W. Influence of hydrophilic co-monomer on the drug release from hydrogels with thermosensitive $\mathrm{N}$-(isopropyl)acrylamide derivatives. J Nanosci Nanotechnol. 2019; 19(5):2514-2521.

3. Serrano DR, Gallagher KH, Healy AM. Emerging nanonisation technologies: Tailoring crystalline versus amorphous nanomaterials. Curr Top Med Chem. 2015:15(22):2327-2340.

4. Jin K, Luo Z, Zhang B, Pang Z. Biomimetic nanoparticles for inflammation targeting. Acta Pharm Sin B. 2018;8(1):23-33.

5. Paranjpe M, Müller-Goymann CC. Nanoparticle-mediated pulmonary drug delivery: A review. Int J Mol Sci. 2014;15(4):5852-5873.

6. Ostróżka-Cieślik A, Sarecka-Hujar B. The use of nanotechnology in modern pharmacotherapy. In: Grumezescu AM. Multifunctional Systems for Combined Delivery, Biosensing and Diagnostics. Amsterdam, the Netherlands: Elsevier Inc.; 2017:139-158.

7. Cicha I, Singh R, Garlichs CD, Alexiou C. Nano-biomaterials for cardiovascular applications: Clinical perspective. J Control Release. 2016; 229:23-36.

8. Swain S, Sahu PK, Beg S, Babu SM. Nanoparticles for cancer targeting: Current and future directions. Curr Drug Deliv. 2016;13(8):1290-1302.

9. Brambilla D, Le Droumaguet B, Nicolas J, et al. Nanotechnologies for Alzheimer's disease: Diagnosis, therapy, and safety issues. Nanomedicine. 2011;7(5):521-540.

10. Torres-Ortega PV, Saludas L, Hanafy AS, Garbayo E, Blanco-Prieto MJ. Micro- and nanotechnology approaches to improve Parkinson's disease therapy. J Control Release. 2019;295:201-213.

11. Oyarzun-Ampuero F, Vidal A, Concha M, Morales J, Orellana S, MorenoVilloslada I. Nanoparticles for the treatment of wounds. Curr Pharm Des. 2015;21(29):4329-4341.

12. Gupta R, Xie H. Nanoparticles in daily life: Applications, toxicity and regulations. J Environ Pathol Toxicol Oncol. 2018;37(3):209-230.

13. Baran A. Nanotechnology: Legal and ethical issues. Economics and Management. 2016;8:47-54.
14. Paradise J. Regulating nanomedicine at the Food and Drug Administration. AMA J Ethics. 2019;21(4):E347-355.

15. Bozzuto G, Molinari A. Liposomes as nanomedical devices. Int J Nanomedicine. 2015;10:975-999.

16. Banerjee SS, Aher N, Patil R, Khandare J. Poly(ethylene glycol)-prodrug conjugates: Concept, design, and applications. J Drug Deliv. 2012;2012:103973.

17. Talegaonkar S, Bhattacharyya A. Potential of lipid nanoparticles (SLNs and NLCs) in enhancing oral bioavailability of drugs with poor intestinal permeability. AAPS Pharm Sci Tech. 2019;20(3):121.

18. Momoh MA, Esimone CO. Phospholipon $90 \mathrm{H}(\mathrm{P} 90 \mathrm{H})$-based PEGylated microscopic lipospheres delivery system for gentamicin: An antibiotic evaluation. Asian Pac J Trop Biomed. 2012;2(11):889-894.

19. Kovács A, Berkó S, Csányi E, Csóka I. Development of nanostructured lipid carriers containing salicyclic acid for dermal use based on the Quality by Design method. Eur J Pharm Sci. 2017;99:246-257.

20. Khosa A, Reddi S, Saha RN. Nanostructured lipid carriers for site-specific drug delivery. Biomed Pharmacother. 2018;103:598-613.

21. Andonova V, Peneva P. Characterization methods for solid lipid nanoparticles (SLN) and nanostructured lipid carriers (NLC). Curr Pharm Des. 2017;23:6630-6642.

22. Couvreur P, Barratt G, Fattal E, Legrand P, Vauthier C. Nanocapsule technology: A review. Crit Rev Ther Drug Carrier Syst. 2002;19(2):99-134.

23. Tripathy S, Das MK. Dendrimers and their applications as novel drug delivery carriers. J Appl Pharmaceut Sci. 2013;3(9):142-149.

24. Palmerston Mendes L, Pan J, Torchilin VP. Dendrimers as nanocarriers for nucleic acid and drug delivery in cancer therapy. Molecules. 2017;22(9). pii: E1401. doi:10.3390/molecules22091401

25. Cai $X$, Hao J, Zhang $X$, et al. The polyhydroxylated fullerene derivative $\mathrm{C} 60(\mathrm{OH}) 24$ protects mice from ionizing-radiation-induced immune and mitochondrial dysfunction. Toxicol Appl Pharmacol. 2010;243(1): 27-34.

26. Ostróżka-Cieślik A, Sarecka-Hujar B. Perspectives of the carbon nanoparticles use in cancer therapy and imaging. Post Biol Kom. 2017;44: 171-184.

27. Chaudhary KT, Rizvi ZH, Bhatti KA, Ali J, Yupapin PP. Multiwalled carbon nanotube synthesis using arc discharge with hydrocarbon as feedstock. J Nanomater. 2013;2013:105145. doi:10.1155/2013/105145

28. Bianco A, Kostarelos K, Partidos C, Prato M. Biomedical applications of functionalised carbon nanotubes. Chem Commun (Camb). 2005;5: 571-577.

29. Bianco A, Kostarelos K, Prato M. Applications of carbon nanotubes in drug delivery. Curr Opin Chem Biol. 2005;9(6):674-679.

30. Palomäki J, Välimäki E, Sund J, et al. Long, needle-like carbon nanotubes and asbestos activate the NLRP3 inflammasome through a similar mechanism. ACS Nano. 2011;5(9):6861-6870.

31. Donaldson K, Poland CA, Murphy FA, MacFarlane M, Chernova T, Schinwald A. Pulmonary toxicity of carbon nanotubes and asbestos: Similarities and differences. Adv Drug Deliv Rev. 2013;65(15):2078-2086.

32. Hoshyar N, Gray S, Han H, Bao G. The effect of nanoparticle size on in vivo pharmacokinetics and cellular interaction. Nanomedicine (Lond). 2016;11(6):673-692.

33. des Rieux A, Fievez V, Garinot M, Schneider YJ, Préat V. Nanoparticles as potential oral delivery systems of proteins and vaccines: A mechanistic approach. J Control Release. 2006;116(1):1-27.

34. Karagkiozaki V, Pappa F, Arvaniti D, Moumkas A, Konstantinou D, Logothetidis $\mathrm{S}$. The melding of nanomedicine in thrombosis imaging and treatment: A review. Future Sci OA. 2016;2(2):FSO113.

35. Korin N, Kanapathipillai M, Matthews BD, et al. Shear-activated nanotherapeutics for drug targeting to obstructed blood vessels. Science. 2012;337(6095):738-742.

36. Wootton DM, Alevriadou BR. The shear stress of busting blood clots. N Engl J Med. 2012;367(14):1361-1363.

37. Dvir T, Bauer M, Schroeder A, et al. Nanoparticles targeting the infarcted heart. Nano Lett. 2011;11(10):4411-4414.

38. Shao M, Yang W, Han G. Protective effects on myocardial infarction model: Delivery of schisandrin B using matrix metalloproteinase-sensitive peptide-modified, PEGylated lipid nanoparticles. Int J Nanomedicine. 2017;12:7121-7130.

39. Ferreira MP, Ranjan S, Correia AM, et al. In vitro and in vivo assessment of heart-homing porous silicon nanoparticles. Biomaterials. 2016;94:93-104. 
40. Tölli MA, Ferreira MP, Kinnunen SM, et al. In vivo biocompatibility of porous silicon biomaterials for drug delivery to the heart. Biomaterials. 2014;35(29):8394-8405.

41. Chan WT, Liu CC, Chiang Chiau JS, et al. In vivo toxicologic study of larger silica nanoparticles in mice. Int J Nanomedicine. 2017;12: 3421-3432.

42. Yu Y, Li Y, Wang W, et al. Acute toxicity of amorphous silica nanoparticles in intravenously exposed ICR mice. PLoS One. 2013;8(4):e61346.

43. Michler RE. Stem cell therapy for heart failure. Methodist Debakey Cardiovasc J. 2013;9(4):187-194.

44. Ma Q, Yang J, Huang X, et al. Poly(lactide-Cc-glycolide)-monomethoxy-poly-(polyethylene glycol) nanoparticles loaded with melatonin protect adipose-derived stem cells transplanted in infarcted heart tissue. Stem Cells. 2018;36(4):540-550.

45. Nakano Y, Matoba T, Tokutome M, et al. Nanoparticle-mediated delivery of irbesartan induces cardioprotection from myocardial ischemia-reperfusion injury by antagonizing monocyte-mediated inflammation. Sci Rep. 2016;6:29601.

46. Qi Q, Lu L, Li H, et al. Spatiotemporal delivery of nanoformulated liraglutide for cardiac regeneration after myocardial infarction. Int J Nanomedicine. 2017;12:4835-4848.

47. Zhang B, Sai Lung P, Zhao S, Chu Z, Chrzanowski W, Li Q. Shape dependent cytotoxicity of PLGA-PEG nanoparticles on human cells. Sci Rep. 2017;7:7315.

48. Miragoli M, Ceriotti $P$, lafisco $M$, et al. Inhalation of peptide-loaded nanoparticles improves heart failure. Sci TransIMed. 2018:10(424). pii: eaan6205. doi:10.1126/scitransImed.aan6205

49. Holohan C, Van Schaeybroeck S, Longley DB, Johnston PG. Cancer drug resistance: An evolving paradigm. Nat Rev Cancer. 2013;13(10): 714-726.

50. Tang Y, Lamberti G, Curran E, Kiani M, Wang B. Development and characterization of a multi-drug resistant Her-2/neu positive breast cancer cell line [abstract]. FASEB J. 2014;28(Suppl):58.6.

51. Jackson SE, Chester JD. Personalised cancer medicine. Int J Cancer. 2015;137(2):262-266.

52. Miyano T, Wijagkanalan W, Kawakami S, Yamashita F, Hashida M. Anionic amino acid dendrimer-trastuzumab conjugates for specific internalization in HER2-positive cancer cells. Mol Pharm. 2010;7(4): 1318-1327.

53. Kulhari H, Pooja D, Shrivastava S, et al. Trastuzumab-grafted PAMAM dendrimers for the selective delivery of anticancer drugs to HER2positive breast cancer. Sci Rep. 2016;6:23179.

54. Zhong Q, Bielski ER, Rodrigues LS, Brown MR, Reineke JJ, da Rocha SR. Conjugation to poly(amidoamine) dendrimers and pulmonary delivery reduce cardiac accumulation and enhance antitumor activity of doxorubicin in lung metastasis. Mol Pharm. 2016;13(7):2363-2675.

55. Bhadra D, Bhadra S, Jain S, Jain NK. A PEGylated dendritic nanoparticulate carrier of fluorouracil. Int J Pharm. 2003;257(1-2):111-124.

56. Zhao X, Qi T, Kong C, et al. Photothermal exposure of polydopamine-coated branched Au-Ag nanoparticles induces cell cycle arrest, apoptosis, and autophagy in human bladder cancer cells. Int J Nanomedicine. 2018;13:6413-6428.

57. Chen ML, He YJ, Chen XW, Wang JH. Quantum dots conjugated with $\mathrm{Fe}_{3} \mathrm{O}_{4}$-filled carbon nanotubes for cancer-targeted imaging and magnetically guided drug delivery. Langmuir. 2012;28(47):1646916476.

58. Shi X, Wang SH, Shen M, et al. Multifunctional dendrimer-modified multiwalled carbon nanotubes: Synthesis, characterization, and in vitro cancer cell targeting and imaging. Biomacromolecules. 2009; 10(7):1744-1750.

59. Chaudhuri P, Paraskar A, Soni S, Mashelkar RA, Sengupta S. Fullerenol-cytotoxic conjugates for cancer chemotherapy. ACS Nano. 2009; 3(9):2505-2514.

60. Torres VM, Srdjenovic B, Jacevic V, Simic VD, Djordjevic A, Simplício AL. Fullerenol $\mathrm{C} 60(\mathrm{OH}) 24$ prevents doxorubicin-induced acute cardiotoxicity in rats. Pharmacol Rep. 2010;62(4):707-718.

61. Sheng J, Ma B, Yang Q, Zhang C, Jiang Z, Borrathybay E. Tailor-made PEG-DA-CUS nanoparticles enriched in tumor with the aid of retro Diels-Alder reaction triggered by their intrinsic photothermal property. Int J Nanomedicine. 2018;13:4291-4302.

62. Yang SC, Zhu JB. Preparation and characterization of camptothecin solid lipid nanoparticles. Drug Dev Ind Pharm. 2002;28(3):265-274.
63. Rodenak-Kladniew B, Islan GA, de Bravo MG, Durán N, Castro GR. Design, characterization and in vitro evaluation of linalool-loaded solid lipid nanoparticles as potent tool in cancer therapy. Colloids Surf B Biointerfaces. 2017;154:123-132.

64. Zhou X, Liu HY, Zhao H, Wang T. RGD-modified nanoliposomes containing quercetin for lung cancer targeted treatment. Onco Targets Ther. 2018;11:5397-5405.

65. Hasan M, Belhaj N, Benachour H, et al. Liposome encapsulation of curcumin: Physico-chemical characterizations and effects on MCF7 cancer cell proliferation. Int J Pharm. 2014;461(1-2):519-528.

66. Chen Q, Liu J. Transferrin and folic acid co-modified bufalin-loaded nanoliposomes: Preparation, characterization, and application in anticancer activity. Int J Nanomedicine. 2018;13:6009-6018.

67. Zabielska-Koczywąs K, Wojtalewicz A, Użarowska E, et al. Distribution of glutathione-stabilized gold nanoparticles in feline fibrosarcomas and their role as a drug delivery system for doxorubicin-preclinical studies in a murine model. Int J Mol Sci. 2018;19(4):1021.

68. Hersh DS, Wadajkar AS, Roberts N, et al. Evolving drug delivery strategies to overcome the blood-brain barrier. Curr Pharm Des. 2016; 22(9):1177-1193.

69. Dordević SM, Radulović TS, Cekić ND, et al. Experimental design in formulation of diazepam nanoemulsions: Physicochemical and pharmacokinetic performances. JPharm Sci. 2013;102(11):4159-4172.

70. Dordević SM, Cekić ND, Savić MM, et al. Parenteral nanoemulsions as promising carriers for brain delivery of risperidone: Design, characterization and in vivo pharmacokinetic evaluation. Int J Pharm. 2015; 493(1-2):40-54.

71. Sadegh Malvajerd S, Azadi A, Izadi Z, et al. Brain delivery of curcumin using solid lipid nanoparticles and nanostructured lipid carriers: Preparation, optimization, and pharmacokinetic evaluation. ACS Chem Neurosci. 2019;10(1):728-739. doi:10.1021/acschemneuro.8b00510

72. Liu S, Ho PC. Intranasal administration of brain-targeted HP- $\beta-C D /$ chitosan nanoparticles for delivery of scutellarin, a compound with protective effect in cerebral ischaemia. J Pharm Pharmacol. 2017; 69(11):1495-1501.

73. Sharma D, Sharma RK, Bhatnagar A, et al. Nose to brain delivery of midazolam loaded PLGA nanoparticles: In vitro and in vivo investigations. Curr Drug Deliv. 2016;13(4):557-564.

74. Erkkinen MG, Kim MO, Geschwind MD. Clinical neurology and epidemiology of the major neurodegenerative diseases. Cold Spring Harb Perspect Biol. 2018;10(4). pii: a033118. doi:10.1101/cshperspect.a033118

75. Serý O, Povová J, Míšek I, Pešák L, Janout V. Molecular mechanisms of neuropathological changes in Alzheimer's disease: A review. Folia Neuropathol. 2013;51(1):1-9.

76. Bartus RT, Emerich DF. Cholinergic markers in Alzheimer disease. JAMA. 1999;282(23):2208-2209.

77. Agatonovic-Kustrin S, Kettle C, Morton DW. A molecular approach in drug development for Alzheimer's disease. Biomed Pharmacother. 2018;106:553-565.

78. Wilson B, Samanta MK, Santhi K, Kumar KP, Paramakrishnan N, Suresh B. Targeted delivery of tacrine into the brain with polysorbate 80 -coated poly(n-butylcyanoacrylate) nanoparticles. Eur J Pharm Biopharm. 2008;70(1):75-84.

79. Tian XH, Lin XN, Wei F, et al. Enhanced brain targeting of temozolomide in polysorbate- 80 coated polybutylcyanoacrylate nanoparticles. Int J Nanomedicine. 2011;6:445-452.

80. Sun $\mathrm{W}$, Xie C, Wang H, Hu Y. Specific role of polysorbate 80 coating on the targeting of nanoparticles to the brain. Biomaterials. 2004; 25(15):3065-3071.

81. Truran S, Weissig V, Madine J, et al. Nanoliposomes protect against human arteriole endothelial dysfunction induced by $\beta$-amyloid peptide. J Cereb Blood Flow Metab. 2016;36(2):405-412.

82. Mourtas S, Canovi M, Zona C, et al. Curcumin-decorated nanoliposomes with very high affinity for amyloid- $\beta 1-42$ peptide. Biomaterials. 2011;32(6):1635-1645.

83. Klementieva $\mathrm{O}$, Aso E, Filippini $\mathrm{D}$, et al. Effect of poly(propylene imine) glycodendrimers on $\beta$-amyloid aggregation in vitro and in APP/PS1 transgenic mice, as a model of brain amyloid deposition and Alzheimer's disease. Biomacromolecules. 2013;14(10):3570-3580.

84. Klajnert $B$, Wasiak $T$, lonov $M$, et al. Dendrimers reduce toxicity of $A \beta$ 1-28 peptide during aggregation and accelerate fibril formation. Nanomedicine. 2012;8(8):1372-1378. 
85. Li H, Luo Y, Derreumaux P, Wei G. Carbon nanotube inhibits the formation of $\beta$-sheet-rich oligomers of the Alzheimer's amyloid- $\beta(16-22)$ peptide. Biophys J. 2011;101(9):2267-2276.

86. Yang Z, Zhang Y, Yang Y, et al. Pharmacological and toxicological target organelles and safe use of single-walled carbon nanotubes as drug carriers in treating Alzheimer disease. Nanomedicine. 2010; 6(3):427-441.

87. Lohan S, Raza K, Mehta SK, Bhatti GK, Saini S, Singh B. Anti-Alzheimer's potential of berberine using surface decorated multi-walled carbon nanotubes: A preclinical evidence. Int J Pharm. 2017;530(1-2): 263-278.

88. Smith Y, Wichmann T, Factor SA, DeLong MR. Parkinson's disease therapeutics: New developments and challenges since the introduction of levodopa. Neuropsychopharmacology. 2012;37(1):213-246.

89. Niu X, Chen J, Gao J. Nanocarriers as a powerful vehicle to overcome blood-brain barrier in treating neurodegenerative diseases: Focus on recent advances. Asian J Pharm Sci. 2019;14(5):480-496.

90. TreatER Project. https://treater.eu/clinical-study/. Accessed March 3 2019.

91. Hu K, Chen X, Chen W, et al. Neuroprotective effect of gold nanoparticles composites in Parkinson's disease model. Nanomedicine. 2018;14(4):1123-1136.

92. Cao X, Hou D, Wang L, et al. Effects and molecular mechanism of chitosan-coated levodopa nanoliposomes on behavior of dyskinesia rats. Biol Res. 2016;49(1):32.

93. Aliakbari F, Mohammad-Beigi H, Rezaei-Ghaleh N, et al. The potential of zwitterionic nanoliposomes against neurotoxic alpha-synuclein aggregates in Parkinson's disease. Nanoscale. 2018;10(19): 9174-9185.

94. Al-Dhubiab BE, Nair AB, Kumria R, Attimarad M, Harsha S. Development and evaluation of buccal films impregnated with selegilineloaded nanospheres. Drug Deliv. 2016;23(7):2154-2162.

95. Milowska K, Grochowina J, Katir N, et al. Viologen-phosphorus dendrimers inhibit a-synuclein fibrillation. Mol Pharm. 2013;10(3): 1131-1137.

96. Milowska K, Szwed A, Mutrynowska M, et al. Carbosilane dendrimers inhibit a-synuclein fibrillation and prevent cells from rotenoneinduced damage. Int J Pharm. 2015;484(1-2):268-275.

97. Parani M, Lokhande G, Singh A, Gaharwar AK. Engineered nanomaterials for infection control and healing acute and chronic wounds. ACS Appl Mater Interfaces. 2016;8(16):10049-10069.

98. Chopra I. The increasing use of silver-based products as antimicrobial agents: A useful development or a cause for concern? J Antimicrob Chemother. 2007;59(4):587-590.

99. Prabhu S, Poulose EK. Silver nanoparticles: Mechanism of antimicrobial action, synthesis, medical applications and toxicity effects. Int Nano Lett. 2012;2:2-10.

100. Larese FF, D'Agostin F, Crosera M, et al. Human skin penetration of silver nanoparticles through intact and damaged skin. Toxicology. 2009;255(1-2):33-37.

101. Ziv-Polat O, Topaz M, Brosh T, Margel S. Enhancement of incisional wound healing by thrombin conjugated iron oxide nanoparticles. Biomaterials. 2010;31(4):741-747.
102. Leu JG, Chen SA, Chen HM, et al. The effects of gold nanoparticles in wound healing with antioxidant epigallocatechin gallate and a-lipoic acid. Nanomedicine. 2012;8(5):767-775.

103. Wang S, Yan C, Zhang $X$, et al. Antimicrobial peptide modification enhances the gene delivery and bactericidal efficiency of gold nanoparticles for accelerating diabetic wound healing. Biomater Sci. 2018;6(10):2757-2772.

104. Mirnejad R, Mofazzal Jahromi M, Al-Musawi S, et al. Curcuminloaded chitosan tripolyphosphate nanoparticles as a safe, natural and effective antibiotic inhibits the infection of Staphylococcus aureus and Pseudomonas aeruginosa in vivo. Iran J Biotechnol. 2014;12(3):e1012.

105. Mofazzal Jahromi MA, Sahandi Zangabad P, Moosavi Basri SM, et al. Nanomedicine and advanced technologies for burns: Preventing infection and facilitating wound healing. Adv Drug Deliv Rev. 2018; 123:33-64.

106. Elmoslemany RM, Abdallah OY, El-Khordagui LK, Khalafallah NM. Propylene glycol liposomes as a topical delivery system for miconazole nitrate: Comparison with conventional liposomes. AAPS PharmSciTech. 2012;13(2):723-731.

107. Kianvash N, Bahador A, Pourhajibagher M, et al. Evaluation of propylene glycol nanoliposomes containing curcumin on burn wound model in rat: Biocompatibility, wound healing, and anti-bacterial effects. Drug Deliv Transl Res. 2017:7(5):654-663.

108. Partoazar A, Kianvash N, Darvishi MH, Nasoohi S, Rezayat SM, Bahador A. Ethosomal curcumin promoted wound healing and reduced bacterial flora in second degree burn in rat. Drug Res (Stuttg). 2016; 66(12):660-665

109. Li C, Zhang X, Huang X, Wang X, Liao G, Chen Z. Preparation and characterization of flexible nanoliposomes loaded with daptomycin, a novel antibiotic, for topical skin therapy. Int J Nanomedicine. 2013;8:1285-1292.

110. Xu HL, Chen PP, ZhuGe DL, et al. Liposomes with silk fibroin hydrogel core to stabilize bFGF and promote the wound healing of mice with deep second-degree scald. Adv Healthc Mater. 2017;6(19). doi:10. 1002/adhm.201700344

111. Kim MH, Seo JH, Kim HM, Jeong HJ. Zinc oxide nanoparticles, a novel candidate for the treatment of allergic inflammatory diseases. Eur J Pharmacol. 2014;738:31-39.

112. Ali SS, Morsy R, El-Zawawy NA, Fareed MF, Bedaiwy MY. Synthesized zinc peroxide nanoparticles ( $\mathrm{ZnO} 2-\mathrm{NPs}$ ): A novel antimicrobial, antielastase, anti-keratinase, and anti-inflammatory approach toward polymicrobial burn wounds. Int J Nanomedicine. 2017;12:6059-6073.

113. Chereddy KK, Her CH, Comune M, et al. PLGA nanoparticles loaded with host defense peptide LL37 promote wound healing. J Control Release. 2014;194:138-147.

114. Sargent LM, Porter DW, Staska LM, et al. Promotion of lung adenocarcinoma following inhalation exposure to multi-walled carbon nanotubes. Part Fibre Toxicol. 2014:11:3.

115. Fukushima S, Kasai T, Umeda Y, Ohnishi M, Sasaki T, Matsumoto M. Carcinogenicity of multi-walled carbon nanotubes: Challenging issue on hazard assessment. J Occup Health. 2018;60(1):10-30. 